\title{
Excitation energy transfer from dye molecules to doped graphene ${ }^{\#}$
}

\author{
R S SWATHI $^{\mathrm{a}}$ and K L SEBASTIAN ${ }^{\mathrm{b}, *}$ \\ ${ }^{a}$ School of Chemistry, Indian Institute of Science Education and Research, Thiruvananthapuram 695 016, India \\ ${ }^{b}$ Department of Inorganic and Physical Chemistry, Indian Institute of Science, Bangalore 560 012, India \\ e-mail: kls@ipc.iisc.ernet.in; klsiisc@gmail.com
}

\begin{abstract}
Recently, we have reported theoretical studies on the rate of energy transfer from an electronically excited molecule to graphene. It was found that graphene is a very efficient quencher of the electronically excited states and that the rate $\propto z^{-4}$. The process was found to be effective up to $30 \mathrm{~nm}$ which is well beyond the traditional FRET limit. In this report, we study the transfer of an amount of energy $\hbar \Omega$ from a dye molecule to doped graphene. We find a crossover of the distance dependence of the rate from $z^{-4}$ to exponential as the Fermi level is increasingly shifted into the conduction band, with the crossover occurring at a shift of the Fermi level by an amount $\hbar \Omega / 2$.
\end{abstract}

Keywords. FRET; graphene; quenching; doping; Dirac cone approximation; Fermi golden rule; K-Point; electron-hole pairs.

\section{Introduction}

Excitation energy transfer involving carbon based materials is interesting due to the fact that using such materials, it is possible to measure distances well beyond the traditional FRET limit. In our earlier papers, we have analysed the process of resonance energy transfer from an excited dye molecule to a sheet of graphene. ${ }^{1-3}$ The rate was evaluated as a function of the distance $z$ of the molecule from the graphene sheet. We have found the process of energy transfer to be very efficient and the rate has a $z^{-4}$ dependence on the distance. Our report was the first study on energy transfer to graphene. Recent experiments that have been performed after our theoretical studies have infact found efficient energy transfer to graphene and the process was found to be useful in identifying graphene flakes both on substrates and in solution. ${ }^{4}$ Quenching by graphene was also found to be useful in obtaining good resonance Raman signals from fluorescent samples, ${ }^{5}$ in fabricating graphene based devices ${ }^{6}$ and in quantitative DNA analysis. ${ }^{7,8}$ We have also studied the process of energy transfer from fluorophores to carbon nanotubes and found a $z^{-5}$ dependence. ${ }^{9}$ Quantum chemical studies on energy transfer involving two carbon nanotubes have also been reported. ${ }^{10}$ All the above studies involve energy transfer to extended charge densities of carbon based materials and hence found a deviation from the $z^{-6}$ dependence, which has been obtained within the

\footnotetext{
\#Dedicated to Prof. N Sathyamurthy on his 60th birthday

*For correspondence
}

dipolar approximation. Such deviations from the dipolar approximation have also been found in polymers, ${ }^{11}$ quantum wells ${ }^{12}$ etc. The Fermi surface of undoped graphene is a set of six points known as the K-points. As a result of this, the density of states at the Fermi level is zero. It is possible to shift the Fermi level of graphene away from the K-point experimentally, either by electrical or chemical doping. ${ }^{13,14}$ This will make the density of states at the new Fermi level non-zero. In this paper, we study the effect of shifting the Fermi level of graphene on the distance dependence of the rate of energy transfer to graphene. ${ }^{15,16}$ We imagine that the Fermi level is shifted into the conduction band to a level with magnitude of wave vector, $k_{F}$. To keep the calculations simple, we use the Dirac cone approximation, which allows us to get analytical expressions for the rate at large distances. We note that as we are shifting the Fermi level by rather large amounts, there will be sizeable corrections to the rate due to deviations from the Dirac cone approximation and hence our conclusions are of qualitative nature.

\section{Model for the rate}

We consider the process of excitation energy transfer from a dye molecule to doped graphene. Since the energy donor (dye molecule) has a localized electronic charge density, we think of the interaction between the donor and the acceptor as that between the transition dipole of the donor, $\boldsymbol{\mu}_{e g}^{D}$, given by $\boldsymbol{\mu}_{e g}^{D}=$ $-e \int d \mathbf{r}_{1} \psi_{e}^{D *}\left(\mathbf{r}_{1}\right) \mathbf{r}_{1} \psi_{g}^{D}\left(\mathbf{r}_{1}\right)$ and the transition charge 
density $\rho\left(\mathbf{r}_{2}\right)=-e \psi_{g}^{A *}\left(\mathbf{r}_{2}\right) \psi_{e}^{A}\left(\mathbf{r}_{2}\right)$ of the acceptor. ${ }^{3,9}$ The energy of interaction is given by

$$
U=\boldsymbol{\mu}_{e g}^{D} \cdot \nabla \Phi,
$$

where $\Phi$ is the electrostatic potential at the point $\mathbf{r}$ (the position of the donor) due to the charge density $\rho\left(\mathbf{r}_{2}\right)$ and is given by

$$
\Phi(\mathbf{r})=\frac{1}{4 \pi \epsilon} \int d \mathbf{r}_{2} \frac{\rho\left(\mathbf{r}_{2}\right)}{\left|\mathbf{r}-\mathbf{r}_{2}\right|} .
$$

As a result of energy transfer, an electron in graphene with wave vector $\mathbf{k}_{i}$ is excited to a level with wave vector $\mathbf{k}_{f}$. We define $\mathbf{k}_{f}=\mathbf{k}_{i}+\mathbf{q}$, where $\mathbf{q} \hbar$ is the momentum transferred to graphene. The rate of energy transfer can be evaluated using the Fermi golden rule and is given by

$$
k=\frac{2 \pi}{\hbar} \sum_{\mathbf{k}_{i}} \sum_{\mathbf{q}}\left|U_{\mathbf{k}_{i}, \mathbf{q}}\right|^{2} \delta\left(E_{\mathbf{k}_{i}+\mathbf{q}}^{+}-E_{\mathbf{k}_{i}}^{-}-\hbar \Omega\right),
$$

where $\hbar \Omega$ is the emission energy of the fluorophore. We use the tight binding wave functions of graphene and evaluate the matrix element (for details, see the References 1-3) using equations (1) and (2) and find it to be

$$
U_{\mathbf{k}_{i}, \mathbf{q}}=\frac{e}{4 \epsilon A}\left[e^{i\left(\delta_{\mathbf{k}_{i}+\mathbf{q}}-\delta_{\mathbf{k}_{i}}\right)}-1\right] \boldsymbol{\mu}_{e g}^{D} \cdot(i \hat{\mathbf{q}}+\hat{\mathbf{k}}) e^{-q z} e^{-i \mathbf{q} \cdot \mathbf{X}},
$$

where $\hat{\mathbf{q}}=\frac{\mathbf{q}}{q}$ is the unit vector in the direction of $\mathbf{q}$ and $\hat{\mathbf{k}}$ is the unit vector in the $z$ direction. We have also used $\mathbf{r}=(\mathbf{X}, z)$, with $\mathbf{X}$ being parallel to the plane of graphene. $A$ is the area of the graphene lattice and

$$
\delta_{\mathbf{k}}=\tan ^{-1}\left(\frac{k_{y}}{k_{x}}\right)=\varphi_{\mathbf{k}},
$$

where $\varphi_{\mathbf{k}}$ is the angle that the vector $\mathbf{k}$ makes with the $\mathrm{x}$-axis. We substitute equation (4) for the interaction energy into equation (3) to get

$$
k=\frac{\pi e^{2}}{4 \hbar \epsilon^{2} A^{2}} \sum_{\mathbf{q}}\left|\boldsymbol{\mu}_{e g}^{D} .(i \hat{\mathbf{q}}+\hat{\mathbf{k}})\right|^{2} \exp (-2 q z) G(\mathbf{q}),
$$

where

$G(\mathbf{q})=\sum_{\mathbf{k}_{\mathbf{i}}}\left[1-\cos \left(\varphi_{\mathbf{k}_{i}+\mathbf{q}}-\varphi_{\mathbf{k}_{i}}\right)\right] \delta\left(E_{\mathbf{k}_{i}+\mathbf{q}}^{+}-E_{\mathbf{k}_{i}}^{-}-\hbar \Omega\right)$.

When the Fermi level of graphene is shifted into the conduction band, the rate of energy transfer has contributions from two different sets of transitions. In the first, $\mathbf{k}_{i}$ lies in the valence band with $0 \leq k_{i} \leq \infty$ and $\mathbf{k}_{f}$ lies in the conduction band with $k_{F}<k_{f}<\infty$. In the second, both $\mathbf{k}_{i}$ and $\mathbf{k}_{f}$ lie in the conduction band with $0 \leq k_{i} \leq k_{F}$ and $k_{F}<k_{f}<\infty$ (see figure 1). The total rate can thus be written as a sum total of both the contributions, $k=k_{1}+k_{2}$. $k_{1}$ and $k_{2}$ are both given by

$$
k_{j}=\frac{\pi e^{2}}{4 \hbar \epsilon^{2} A^{2}} \sum_{\mathbf{q}}\left|\boldsymbol{\mu}_{e g}^{D} \cdot(i \hat{\mathbf{q}}+\hat{\mathbf{k}})\right|^{2} \exp (-2 q z) G_{j}(\mathbf{q}),
$$

but with differing expressions for $G_{j}(\mathbf{q})$.

$$
\begin{aligned}
G_{1}(\mathbf{q})= & \sum_{\substack{\mathbf{k}_{\mathbf{i}} \in \text { valence band }\\
}}\left[1-\cos \left(\varphi_{\mathbf{k}_{i}+\mathbf{q}}-\varphi_{\mathbf{k}_{i}}\right)\right] \\
& \times \delta\left(E_{\mathbf{k}_{i}+\mathbf{q}}^{+}-E_{\mathbf{k}_{i}}^{-}-\hbar \Omega\right) \Theta\left(\left|\mathbf{k}_{i}+\mathbf{q}\right|-k_{F}\right) .
\end{aligned}
$$

The theta function is introduced to satisfy the condition, $k_{F}<k_{f}<\infty$. In a similar fashion,

$$
\begin{aligned}
& G_{2}(\mathbf{q})=\sum_{\mathbf{k}_{\mathbf{i}} \in \text { conduction band }}\left[1+\cos \left(\varphi_{\mathbf{k}_{i}+\mathbf{q}}-\varphi_{\mathbf{k}_{i}}\right)\right] \\
& \times \delta\left(E_{\mathbf{k}_{i}+\mathbf{q}}^{+}-E_{\mathbf{k}_{i}}^{+}-\hbar \Omega\right) \\
& \times \Theta\left(\left|\mathbf{k}_{i}+\mathbf{q}\right|-k_{F}\right) \Theta\left(k_{F}-\left|\mathbf{k}_{i}\right|\right) \text {. }
\end{aligned}
$$

The two theta functions satisfy the conditions, $0 \leq k_{i} \leq$ $k_{F}$ and $k_{F}<k_{f}<\infty$. We now evaluate $G_{1}(\mathbf{q})$ and $G_{2}(\mathbf{q})$, separately. We replace the sum over $\mathbf{k}_{i}$ in the expression for $G_{1}(\mathbf{q})$ by an integral and use the linear dispersion relation for the energy levels of graphene $\left(E_{\mathbf{k}}^{ \pm}= \pm v_{f} k\right)$ to get

$$
\begin{aligned}
G_{1}(\mathbf{q})= & \frac{A}{4 \pi^{2} v_{f}} \int d \mathbf{k}_{i}\left[1-\frac{\mathbf{k}_{i} \cdot\left(\mathbf{k}_{i}+\mathbf{q}\right)}{\left|\mathbf{k}_{i}\right|\left|\left(\mathbf{k}_{i}+\mathbf{q}\right)\right|}\right] \\
& \times \delta\left(\left|\mathbf{k}_{i}\right|+\left|\mathbf{k}_{i}+\mathbf{q}\right|-\frac{\hbar \Omega}{v_{f}}\right) \\
& \times \Theta\left(\left|\mathbf{k}_{i}+\mathbf{q}\right|-k_{F}\right) .
\end{aligned}
$$

\section{Effect of doping on the rate}

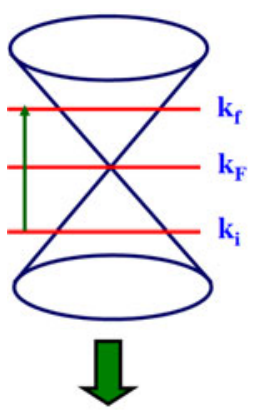

Fermi level at the K-point

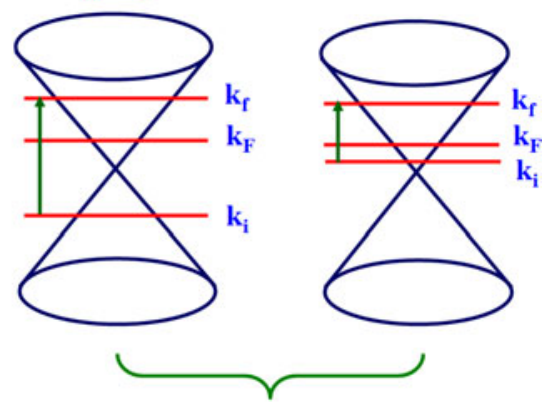

When Fermi level is shifted into the conduction band
Figure 1. A schematic of the energy bands, showing the Fermi wave vectors, the initial and the final wave vectors corresponding to excitation energy transfer. $E_{F}=v_{f} k_{F}$ is the location of the new Fermi level on doping graphene. 
Introducing a new variable $\mathbf{k}_{i}^{\prime}$ defined by $\mathbf{k}_{i}^{\prime}=\mathbf{k}_{i}+\frac{\mathbf{q}}{2}$ leads to

$$
\begin{aligned}
G_{1}(\mathbf{q})= & \frac{A}{4 \pi^{2} v_{f}} \int d \mathbf{k}_{i}^{\prime}\left[1-\frac{\left(\mathbf{k}_{i}^{\prime}-\frac{\mathbf{q}}{2}\right) \cdot\left(\mathbf{k}_{i}^{\prime}+\frac{\mathbf{q}}{2}\right)}{\left|\mathbf{k}_{i}^{\prime}-\frac{\mathbf{q}}{2}\right|\left|\mathbf{k}_{i}^{\prime}+\frac{\mathbf{q}}{2}\right|}\right] \\
& \times \delta\left(\left|\mathbf{k}_{i}^{\prime}-\frac{\mathbf{q}}{2}\right|+\left|\mathbf{k}_{i}^{\prime}+\frac{\mathbf{q}}{2}\right|-\frac{\hbar \Omega}{v_{f}}\right) \\
& \times \Theta\left(\left|\mathbf{k}_{i}^{\prime}+\frac{\mathbf{q}}{2}\right|-k_{F}\right) .
\end{aligned}
$$

We choose the direction of $\mathbf{q}$ as the $\mathrm{x}$-axis and then make another change of variable to $\mathbf{r}$ given by $\mathbf{r}=\frac{\mathbf{k}_{i}^{\prime}}{q / 2}$ to get

$$
\begin{aligned}
G_{1}(\mathbf{q})= & \frac{A q^{2}}{16 \pi^{2} v_{f}} \int d \mathbf{r}\left[1-\frac{(\mathbf{r}-\hat{\mathbf{i}}) \cdot(\mathbf{r}+\hat{\mathbf{i}})}{|\mathbf{r}-\hat{\mathbf{i}}||\mathbf{r}+\hat{\mathbf{i}}|}\right] \\
& \times \delta\left[\frac{q}{2}(|\mathbf{r}-\hat{\mathbf{i}}|+|\mathbf{r}+\hat{\mathbf{i}}|)-\frac{\hbar \Omega}{v_{f}}\right] \\
& \times \Theta\left(\frac{q}{2}|\mathbf{r}+\hat{\mathbf{i}}|-k_{F}\right) .
\end{aligned}
$$

Using $\mathbf{r} \equiv(x, y)$, the above equation can be rewritten as

$$
\begin{aligned}
G_{1}(\mathbf{q})= & \frac{A q^{2}}{8 \pi^{2} v_{f}} \int_{-\infty}^{\infty} d x \int_{0}^{\infty} d y \\
\times & {\left[1-\frac{x^{2}+y^{2}-1}{\sqrt{(x-1)^{2}+y^{2}} \sqrt{(x+1)^{2}+y^{2}}}\right] } \\
& \times \delta\left[\frac { q } { 2 } \left(\sqrt{(x-1)^{2}+y^{2}}\right.\right. \\
& \times \Theta\left(\frac{q}{2} \sqrt{(x+1)^{2}+y^{2}}-k_{F}\right) .
\end{aligned}
$$

We now change over to elliptic coordinates defined by $x=\mu v$ and $y=\sqrt{\left(\mu^{2}-1\right)\left(1-v^{2}\right)}$. The transformation gives $d x d y=\frac{\mu^{2}-v^{2}}{\sqrt{\left(\mu^{2}-1\right)\left(1-v^{2}\right)}} d \mu d v$. With the above transformation, we get

$$
\begin{aligned}
G_{1}(\mathbf{q})= & \frac{A q^{2}}{4 \pi^{2} v_{f}} \int_{1}^{\infty} d \mu \int_{-1}^{1} d v \sqrt{\frac{1-v^{2}}{\mu^{2}-1}} \\
& \times \delta\left(q \mu-\frac{\hbar \Omega}{v_{f}}\right) \Theta\left[\frac{q}{2}(\mu+v)-k_{F}\right] .
\end{aligned}
$$

The integral over $\mu$ can be easily performed to get

$$
\begin{aligned}
G_{1}(\mathbf{q})= & \frac{A q^{2}}{4 \pi^{2}} \frac{\Theta\left(\hbar \Omega-q v_{f}\right)}{\sqrt{(\hbar \Omega)^{2}-q^{2} v_{f}^{2}}} \int_{-1}^{1} d v \sqrt{1-v^{2}} \\
& \times \Theta\left[\frac{q}{2}\left(\frac{\hbar \Omega}{q v_{f}}+v\right)-k_{F}\right] .
\end{aligned}
$$

The above equation can be rewritten as

$$
\begin{aligned}
G_{1}(\boldsymbol{q})= & \frac{A q^{2}}{4 \pi^{2}} \frac{\Theta\left(\hbar \Omega-q v_{f}\right)}{\sqrt{(\hbar \Omega)^{2}-q^{2} v_{f}^{2}}} \Theta\left[1-\frac{2 E_{F}-\hbar \Omega}{q v_{f}}\right] \\
& \times \int_{\operatorname{Max}\left[-1, \frac{2 E_{F}-\hbar \Omega}{q v_{f}}\right]}^{1} d v \sqrt{1-v^{2}} .
\end{aligned}
$$

We now substitute back the above expression into the rate expression of equation (8) and convert the sum over $\boldsymbol{q}$ to an integral to get

$$
\begin{aligned}
k_{1}= & \frac{e^{2}}{64 \hbar \epsilon^{2} \pi^{3}} \int_{0}^{\infty} d q q^{3} e^{-2 q z} \frac{\Theta\left(\hbar \Omega-q v_{f}\right)}{\sqrt{(\hbar \Omega)^{2}-q^{2} v_{f}^{2}}} \\
& \Theta\left[1-\frac{2 E_{F}-\hbar \Omega}{q v_{f}}\right] \int_{\operatorname{Max}\left[-1, \frac{2 E_{F}-\hbar \Omega}{q v_{f}}\right]}^{1} d v \sqrt{1-v^{2}} \\
& \times \int_{0}^{2 \pi} d \theta\left|\boldsymbol{\mu}_{e g}^{D} \cdot(i \hat{\boldsymbol{q}}+\hat{\boldsymbol{k}})\right|^{2}
\end{aligned}
$$

where $(q, \theta)$ are the polar coordinates of $\boldsymbol{q}$. After performing the integral over $\theta$, we average over all possible orientations of the donor transition dipole $\left(\mathrm{see}^{2,3}\right)$ to get

$$
\begin{aligned}
k_{1}= & \frac{e^{2} \mu_{e g}^{2}}{48 \hbar \epsilon^{2} \pi^{2}} \int_{0}^{\infty} d q q^{3} e^{-2 q z} \frac{\Theta\left(\hbar \Omega-q v_{f}\right)}{\sqrt{(\hbar \Omega)^{2}-q^{2} v_{f}^{2}}} \\
\Theta & {\left[1-\frac{2 E_{F}-\hbar \Omega}{q v_{f}}\right] \int_{\operatorname{Max}\left[-1, \frac{2 E_{F}-\hbar \Omega}{q v_{f}}\right]}^{1} d v \sqrt{1-v^{2}} . }
\end{aligned}
$$

Evaluation of the integral over $v$ leads to

$$
\begin{aligned}
k_{1}= & \frac{e^{2} \mu_{e g}^{2}}{96 \hbar \epsilon^{2} \pi^{2}} \int_{0}^{\infty} d q q^{3} e^{-2 q z} \frac{\Theta\left(\hbar \Omega-q v_{f}\right)}{\sqrt{(\hbar \Omega)^{2}-q^{2} v_{f}^{2}}} \\
& \Theta\left[1-\frac{2 E_{F}-\hbar \Omega}{q v_{f}}\right] \\
& \times\left[\frac{\pi}{2}-\left(u \sqrt{1-u^{2}}+\sin ^{-1} u\right)\right],
\end{aligned}
$$

where $u$ is defined by $u=\operatorname{Max}\left[-1, \frac{2 E_{F}-\hbar \Omega}{q v_{f}}\right]$. 
We now evaluate $G_{2}(\boldsymbol{q})$, defined by equation (10). Using the same procedure as before, we find that

$$
\begin{aligned}
G_{2}(\mathbf{q})= & \frac{A q^{2}}{4 \pi^{2} v_{f}} \int_{1}^{\infty} d \mu \int_{-1}^{1} d v \sqrt{\frac{\mu^{2}-1}{1-v^{2}}} \\
& \delta\left(q v-\frac{\hbar \Omega}{v_{f}}\right) \Theta\left[\frac{q}{2}(\mu+v)-k_{F}\right] \\
& \times \Theta\left[k_{F}-\frac{q}{2}(\mu-v)\right] .
\end{aligned}
$$

The integral over $v$ can be easily performed to get

$$
\begin{aligned}
G_{2}(\mathbf{q})= & \frac{A q^{2}}{4 \pi^{2}} \frac{\Theta\left(q v_{f}-\hbar \Omega\right)}{\sqrt{q^{2} v_{f}^{2}-(\hbar \Omega)^{2}}} \int_{1}^{\infty} d \mu \sqrt{\mu^{2}-1} \\
& \times \Theta\left[\frac{q}{2}\left(\frac{\hbar \Omega}{q v_{f}}+\mu\right)-k_{F}\right] \\
& \times \Theta\left[k_{F}-\frac{q}{2}\left(\mu-\frac{\hbar \Omega}{q v_{f}}\right)\right] .
\end{aligned}
$$

The above equation can be rewritten as

$$
\begin{aligned}
G_{2}(\mathbf{q})= & \frac{A q^{2}}{4 \pi^{2}} \frac{\Theta\left(q v_{f}-\hbar \Omega\right)}{\sqrt{q^{2} v_{f}^{2}-(\hbar \Omega)^{2}}} \\
& \times \int_{\operatorname{Max}\left[1, \frac{2 E_{F}-\hbar \Omega}{q v_{f}}\right]}^{\frac{2 E_{F}+\hbar \Omega}{q_{f}}} d \mu \sqrt{\mu^{2}-1} \\
& \times \Theta\left[\frac{2 E_{F}+\hbar \Omega}{q v_{f}}-1\right] .
\end{aligned}
$$

Substituting the above expression back into the rate expression of equation (8) gives

$$
\begin{aligned}
k_{2}= & \frac{e^{2}}{64 \hbar \epsilon^{2} \pi^{3}} \int_{0}^{\infty} d q q^{3} e^{-2 q z} \frac{\Theta\left(q v_{f}-\hbar \Omega\right)}{\sqrt{q^{2} v_{f}^{2}-(\hbar \Omega)^{2}}} \\
& \Theta\left[\frac{2 E_{F}+\hbar \Omega}{q v_{f}}-1\right] \int_{\operatorname{Max}\left[1, \frac{2 E_{F}-\hbar \Omega}{q v_{f}}\right]}^{\frac{2 E_{F}+\hbar \Omega}{q v_{f}}} d \mu \sqrt{\mu^{2}-1} \\
& \times \int_{0}^{2 \pi} d \theta\left|\boldsymbol{\mu}_{e g}^{D} \cdot(i \hat{\mathbf{q}}+\hat{\mathbf{k}})\right|^{2} .
\end{aligned}
$$

The $\theta$ integral can be performed easily, followed by an averaging over all possible orientations of the donor transition dipole to get

$$
\begin{aligned}
k_{2}= & \frac{e^{2} \mu_{e g}^{2}}{48 \hbar \epsilon^{2} \pi^{2}} \int_{0}^{\infty} d q q^{3} e^{-2 q z} \frac{\Theta\left(q v_{f}-\hbar \Omega\right)}{\sqrt{q^{2} v_{f}^{2}-(\hbar \Omega)^{2}}} \\
& \times \Theta\left[\frac{2 E_{F}+\hbar \Omega}{q v_{f}}-1\right] \int_{\operatorname{Max}\left[1, \frac{2 E_{F}-\hbar \Omega}{q v_{f}}\right]}^{\frac{2 E_{F}+\hbar \Omega}{q v_{f}}} d \mu \sqrt{\mu^{2}-1} .
\end{aligned}
$$

The integral over $\mu$ can be evaluated to get

$$
\begin{aligned}
k_{2}= & \frac{e^{2} \mu_{e g}^{2}}{96 \hbar \epsilon^{2} \pi^{2}} \int_{0}^{\infty} d q q^{3} e^{-2 q z} \frac{\Theta\left(q v_{f}-\hbar \Omega\right)}{\sqrt{q^{2} v_{f}^{2}-(\hbar \Omega)^{2}}} \\
& \Theta\left[\frac{2 E_{F}+\hbar \Omega}{q v_{f}}-1\right] \\
& \times\left(-r \sqrt{r^{2}-1}+s \sqrt{s^{2}-1}+\right. \\
& \left.\log \left[r+\sqrt{r^{2}-1}\right]-\log \left[s+\sqrt{s^{2}-1}\right]\right),
\end{aligned}
$$

where $r=\operatorname{Max}\left[1, \frac{2 E_{F}-\hbar \Omega}{q v_{f}}\right]$ and $s=\frac{2 E_{F}+\hbar \Omega}{q v_{f}}$. The integrals over $q$ in the expressions for $k_{1}$ and $k_{2}$ can be performed numerically, thus getting the total rate of transfer when the Fermi level in graphene is shifted into the conduction band.

\section{Large $z$ behaviour of $k_{1}$ and $k_{2}$}

It is easy to analyse the large $z$ behaviour of $k_{1}$ and $k_{2}$ (see the Appendix A for the detailed analysis). When $E_{F}<\frac{\hbar \Omega}{2}$ and $z>\frac{v_{f}}{2 \Delta \epsilon}$, with $\Delta \epsilon=\frac{\hbar \Omega}{2}-E_{F}$ and $\hbar \Omega>>\Delta \epsilon$, the long-range behaviour of $k_{1}$ is given by equation (32) of the Appendix A as

$$
k_{1}=\frac{e^{2} \mu_{e g}^{2}}{256 \pi \Omega \hbar^{2} \epsilon^{2} z^{4}} .
$$

In the case when $E_{F}>\frac{\hbar \Omega}{2}$ and $z>\frac{v_{f}}{4 \Delta \epsilon}$, with $\hbar \Omega>\Delta \epsilon$,

$$
k_{1}=\frac{e^{2} \mu_{e g}^{2}|\Delta \epsilon|^{3 / 2}}{96 \sqrt{2} \Omega \hbar^{2} \epsilon^{2} \pi^{3 / 2} v_{f}^{3 / 2} z^{5 / 2}} e^{\frac{-4|\Delta \epsilon| z}{v_{f}}},
$$

as obtained in equation (39) of the Appendix A. The major contribution from the $k_{1}$ term to the rate comes only when $E_{F}<\frac{\hbar \Omega}{2}$ and it has a power law dependence $\left(z^{-4}\right)$ on the distance. When $E_{F}>\frac{\hbar \Omega}{2}$, the contribution from $k_{1}$ decreases exponentially with $z$ and hence 
is very small. The long-range behaviour of $k_{2}$ for both $E_{F}<\frac{\hbar \Omega}{2}$ and $E_{F}>\frac{\hbar \Omega}{2}$ is given by equations (44) and (49) of the Appendix B as

$$
\begin{aligned}
k_{2}= & \frac{1}{192 \sqrt{z}}\left[\frac{e^{4} \mu_{e g}^{4} \hbar^{3} \Omega^{5}}{\pi^{3} \epsilon^{4} v_{f}^{7}}\right]^{\frac{1}{2}} e^{\frac{-2 z \hbar \Omega}{v_{f}}} \\
& \times\left(r \sqrt{r^{2}-1}-\log \left[r+\sqrt{r^{2}-1}\right]\right),
\end{aligned}
$$

where $r=1+\frac{2 E_{F}}{\hbar \Omega}$.

Therefore, the large $z$ behaviour of $k_{2}$ is exponential. Thus, in the case when $E_{F}<\frac{\hbar \Omega}{2}$, the rate of transfer to doped graphene has a power law dependence on the distance (arising from the $k_{1}$ term), while when $E_{F}>\frac{\hbar \Omega}{2}$, the rate has an exponential dependence (arising due to both $k_{1}$ and $k_{2}$ terms). Therefore, as the Fermi level is increasingly shifted into the conduction band, there is a crossover of the distance dependence of the rate from power law to exponential and the crossover occurs over a region of $E_{F}$ centred at $\frac{\hbar \Omega}{2}$.

\section{Results}

We performed numerical calculations for evaluating the rates of energy transfer from a fluorophore to doped graphene. Note that the numerical values of the rates, $k_{1}$ and $k_{2}$, obtained using the equations derived in the earlier sections have been multiplied by a factor of 2 to account for the two inequivalent K-points of graphene. We take the emission energy of the fluorophore, $\hbar \Omega$ to

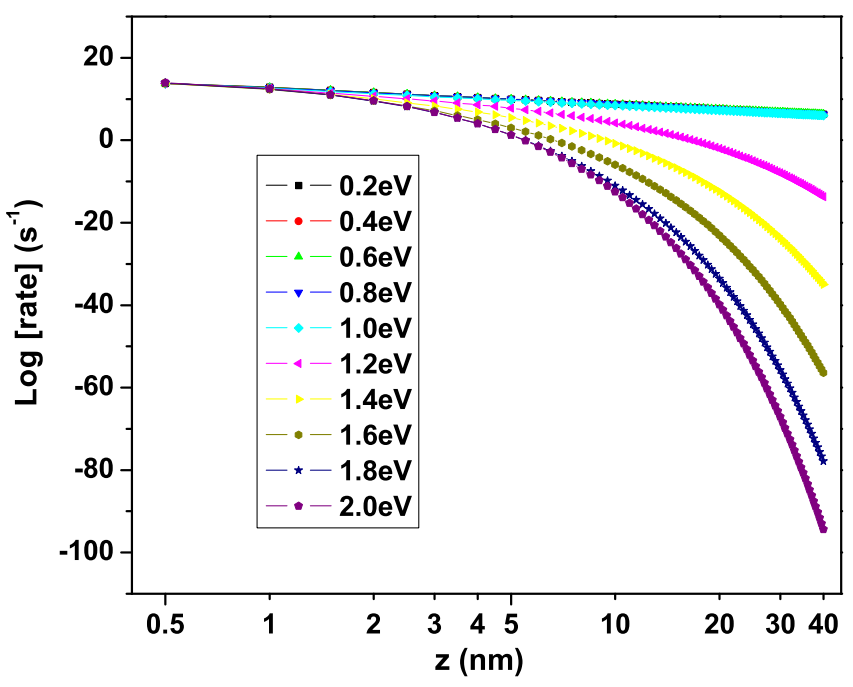

Figure 2. The rate of energy transfer as a function of distance, as the Fermi level is shifted into the conduction band. The emission energy of the fluorophore is taken to be $\hbar \Omega=$ $2.0 \mathrm{eV}$.

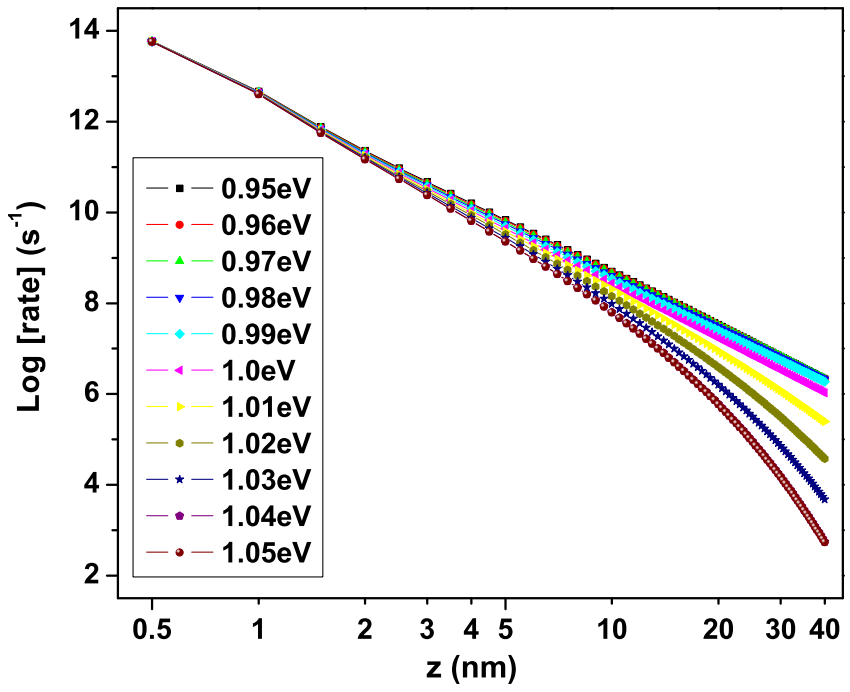

Figure 3. The rate of energy transfer as a function of distance, as the Fermi level is shifted into the conduction band (a closer look at the crossover region). The emission energy of the fluorophore is taken to be $\hbar \Omega=2.0 \mathrm{eV}$.

be $2.0 \mathrm{eV}$. Such low energy emission has been found in squarylium dyes. ${ }^{17}$ We take $\mu_{e g}=4.5 \mathrm{D}$. Figure 2 shows a plot of the logarithm of the rate as a function of the logarithm of the distance as the Fermi level is shifted increasingly into the conduction band in the range $0.2-2.0 \mathrm{eV}$. The log-log plot is linear showing that the rate has a power law dependence $\left(z^{-4}\right)$ on the distance for $E_{F}<1.0 \mathrm{eV}$. After around $1.0 \mathrm{eV}$, there is deviation from linearity in the log-log plot and

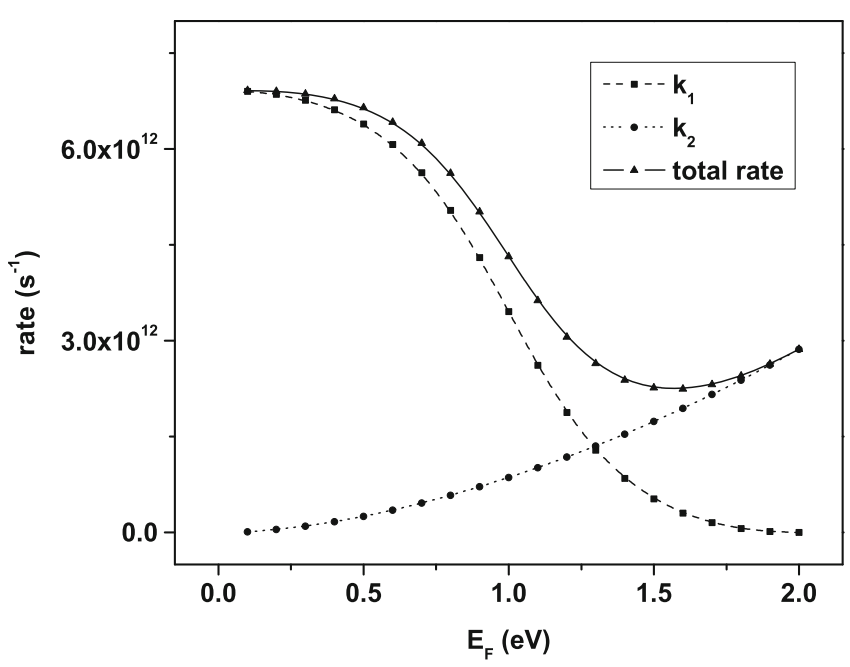

Figure 4. The rate of energy transfer as a function of the Fermi energy of graphene, at a distance of $10 \AA$. The figure shows plots of $k_{1}$ and $k_{2}$ separately along with the total rate of transfer. 
the dependence becomes exponential. In order to look into the crossover region more closely, in figure 3, we show a plot of the rate as the Fermi level is moved into the conduction band in the range $0.95-1.05 \mathrm{eV}$. This clearly shows that there is a crossover of the distance dependence of the rate from power law to exponential as the Fermi level is increasingly shifted into the conduction band. It should be possible to observe this effect experimentally.

Figure 4 shows a plot of the $k_{1}$ and $k_{2}$ terms, and the total rate of transfer as a function of the Fermi energy of graphene at a fixed distance, $z=10 \AA$. The contribution from the $k_{1}$ term decreases as the Fermi level is increasingly shifted into the conduction band, while that from the $k_{2}$ term increases. From the figure, it is clear that the total rate is governed mainly by the $k_{1}$ term up to the crossover point. Beyond that, the contribution from $k_{1}$ term is small and the total rate is governed by the $k_{2}$ term. This is clearly a density of states effect.

Finally, we mention a closely related work on energy transfer to graphene that appeared recently after we finished our work, ${ }^{15}$ wherein the authors consider the process of energy transfer to the electron-hole pairs and the plasmons of graphene. ${ }^{18}$ The results obtained in $^{18}$ are in agreement with our earlier calculations on energy transfer to the electron-hole pairs of undoped graphene $^{1-3}$ and current calculations on transfer to the electron-hole pairs of doped graphene. ${ }^{15,16}$ However, they also study the possibility of collective excitations in graphene (plasmonic excitations), as a result of energy transfer.

\section{Conclusions}

We have studied the process of energy transfer from a fluorophore to doped graphene. We have analysed the distance dependence of the rate of transfer as the Fermi level of graphene is shifted away from the K-point into the conduction band. We find a crossover of the dependence from power law $\left(z^{-4}\right)$ to exponential as the Fermi level is increasingly moved into the conduction band. The point of crossover is at a shift of the Fermi level by $\hbar \Omega / 2$.

\section{Acknowledgements}

RSS acknowledges the Council of Scientific and Industrial Research (CSIR), India and Bristol-Myers-Squibb Fellowship for financial support. The work of KLS was supported by the J C Bose Fellowship of the Department of Science and Technology (DST), India.

\section{Appendix A}

\section{Behaviour of rate constant, $k_{1}$}

We first look at $k_{1}$. We consider two separate cases.

1. Case I: We consider the case where $E_{F}$ is close to $\frac{\hbar \Omega}{2}$, but less than it. We put $\Delta \epsilon=\frac{\hbar \Omega}{2}-E_{F} . k_{1}$ is then given by

$$
\begin{aligned}
k_{1}= & \frac{e^{2} \mu_{e g}^{2}}{48 \hbar \epsilon^{2} \pi^{2}} \int_{0}^{\infty} d q q^{3} e^{-2 q z} \frac{\Theta\left(\hbar \Omega-q v_{f}\right)}{\sqrt{(\hbar \Omega)^{2}-q^{2} v_{f}^{2}}} \\
& \times \Theta\left[1+\frac{2 \Delta \epsilon}{q v_{f}}\right] \int_{\operatorname{Max}\left[-1,-\frac{2 \Delta \epsilon}{q_{f}}\right]}^{1} d v \sqrt{1-v^{2}} .
\end{aligned}
$$

As $\Delta \epsilon>0, \Theta\left[1+\frac{2 \Delta \epsilon}{q v_{f}}\right]=1$. For $z>\frac{v_{f}}{2 \Delta \epsilon}$, the major contribution to the above integral is from $q \in$ $\left(0, \frac{\Delta \epsilon}{v_{f}}\right)$. In this range,

$$
\int_{\operatorname{Max}\left[-1,-\frac{2 \Delta \epsilon}{q v_{f}}\right]}^{1} d v \sqrt{1-v^{2}}=\int_{-1}^{1} d v \sqrt{1-v^{2}}=\frac{\pi}{2} .
$$

If $\frac{\hbar \Omega}{2}>>\Delta \epsilon$, then, in this range, $(\hbar \Omega)^{2}-q^{2} v_{f}^{2} \simeq$ $(\hbar \Omega)^{2}$ and the integral in equation (30) may be approximated as

$$
k_{1}=\frac{e^{2} \mu_{e g}^{2}}{96 \pi \hbar^{2} \epsilon^{2} \Omega} \int_{0}^{\infty} d q q^{3} e^{-2 q z}=\frac{e^{2} \mu_{e g}^{2}}{256 \pi \Omega \hbar^{2} \epsilon^{2} z^{4}} .
$$

2. Case II: We now consider the case $E_{F}>\frac{\hbar \Omega}{2}$. Hence, $\frac{\hbar \Omega}{2}-E_{F}=-|\Delta \epsilon|$. Then,

$$
\begin{aligned}
k_{1}= & \frac{e^{2} \mu_{e g}^{2}}{48 \hbar \epsilon^{2} \pi^{2}} \int_{0}^{\infty} d q q^{3} e^{-2 q z} \frac{\Theta\left(\hbar \Omega-q v_{f}\right)}{\sqrt{(\hbar \Omega)^{2}-q^{2} v_{f}^{2}}} \\
& \times \Theta\left[1-\frac{2|\Delta \epsilon|}{q v_{f}}\right] \int_{\operatorname{Max}\left[-1, \frac{2|\Delta \epsilon|}{q v_{f}}\right]}^{1} d v \sqrt{1-v^{2}} .
\end{aligned}
$$

For large $z\left(z>>\frac{v_{f}}{4|\Delta \epsilon|}\right)$, this may be approximated as

$$
\begin{aligned}
k_{1}= & \frac{e^{2} \mu_{e g}^{2}}{48 \hbar \epsilon^{2} \pi^{2}} \int_{\frac{2|\Delta \epsilon|}{v_{f}}}^{\infty} d q q^{3} e^{-2 q z} \frac{\Theta\left(\hbar \Omega-q v_{f}\right)}{\sqrt{(\hbar \Omega)^{2}-q^{2} v_{f}^{2}}} \\
& \times \int_{\frac{2|\Delta \epsilon|}{q v_{f}}}^{1} d v \sqrt{1-v^{2}} .
\end{aligned}
$$


For $z$ such that $\frac{4|\Delta \epsilon| z}{v_{f}}>>1$, in the above integral over $q$, the contribution is from $q$ in the vicinity of $\frac{2|\Delta \epsilon|}{v_{f}}$. We now change the variable of integration from $q$ to $y$ defined by $q=\frac{2|\Delta \epsilon| y}{v_{f}}$. Using the above transformation, $k_{1}$ is given by

$$
\begin{aligned}
k_{1}= & \frac{e^{2} \mu_{e g}^{2}|\Delta \epsilon|^{4}}{3 \hbar \epsilon^{2} \pi^{2} v_{f}^{4}} \int_{1}^{\infty} d y y^{3} e^{\frac{-4|\Delta \epsilon| z y}{v_{f}}} \\
& \times \frac{\Theta(\hbar \Omega-2|\Delta \epsilon| y)}{\sqrt{(\hbar \Omega)^{2}-4|\Delta \epsilon|^{2} y^{2}}} \int_{\frac{1}{y}}^{1} d v \sqrt{1-v^{2}} .
\end{aligned}
$$

Now, the major contribution to the above integral comes from values of $y \simeq 1$. If $y$ is close to unity,

$$
\int_{\frac{1}{y}}^{1} d v \sqrt{1-v^{2}} \simeq \frac{2 \sqrt{2}}{3}\left(\frac{y-1}{y}\right)^{3 / 2} .
$$

Hence,

$$
\begin{aligned}
k_{1} \simeq & \frac{2 \sqrt{2} e^{2} \mu_{e g}^{2}|\Delta \epsilon|^{4}}{9 \hbar \epsilon^{2} \pi^{2} v_{f}^{4}} \int_{1}^{\infty} d y y^{3 / 2}(y-1)^{3 / 2} e^{\frac{-4|\Delta \epsilon| z y}{v_{f}}} \\
& \times \frac{\Theta(\hbar \Omega-2|\Delta \epsilon| y)}{\sqrt{(\hbar \Omega)^{2}-4|\Delta \epsilon|^{2} y^{2}}}
\end{aligned}
$$

For $\frac{\hbar \Omega}{2}>>|\Delta \epsilon|$ and $y$ in the vicinity of $1, \Theta(\hbar \Omega-$ $2|\Delta \epsilon| y)=1$ and $\sqrt{(\hbar \Omega)^{2}-4|\Delta \epsilon|^{2} y^{2}} \simeq \hbar \Omega$. The integral over $y$ can now be evaluated to get:

$$
k_{1} \simeq \frac{e^{2} \mu_{e g}^{2}|\Delta \epsilon|^{2}}{48 \sqrt{2} \Omega \hbar^{2} \epsilon^{2} \pi^{2} v_{f}^{2} z^{2}} e^{\frac{-2|\Delta \epsilon| z}{v_{f}}} K_{2}\left(\frac{2|\Delta \epsilon| z}{v_{f}}\right) .
$$

For large values of $z$, the asymptotic form of the Bessel function $K_{2}\left(\frac{2|\Delta \epsilon| z}{v_{f}}\right)$ is given by $K_{2}\left(\frac{2|\Delta \epsilon| z}{v_{f}}\right) \simeq$ $\sqrt{\frac{\pi v_{f}}{4|\Delta \epsilon| z}} e^{\frac{-2|\Delta \epsilon| z}{v_{f}}}$. Therefore, we get

$$
k_{1} \simeq \frac{e^{2} \mu_{e g}^{2}|\Delta \epsilon|^{3 / 2}}{96 \sqrt{2} \Omega \hbar^{2} \epsilon^{2} \pi^{3 / 2} v_{f}^{3 / 2} z^{5 / 2}} e^{\frac{-4|\Delta \epsilon| z}{v_{f}}} .
$$

\section{Appendix B}

Behaviour of rate constant, $k_{2}$

We now analyse $k_{2}$. As before, we consider two separate cases.

1. Case I: We consider the case $E_{F}<\frac{\hbar \Omega}{2}$. Therefore, $2 E_{F}-\hbar \Omega<0$ and hence $k_{2}$ is given by

$$
\begin{aligned}
k_{2}= & \frac{e^{2} \mu_{e g}^{2}}{48 \hbar \epsilon^{2} \pi^{2}} \int_{0}^{\infty} d q q^{3} e^{-2 q z} \frac{\Theta\left(q v_{f}-\hbar \Omega\right)}{\sqrt{q^{2} v_{f}^{2}-(\hbar \Omega)^{2}}} \\
& \times \int_{1}^{\frac{2 E_{F}+\hbar \Omega}{q v_{f}}} d \mu \sqrt{\mu^{2}-1} \Theta\left[\frac{2 E_{F}+\hbar \Omega}{q v_{f}}-1\right] .
\end{aligned}
$$

Using the two theta functions in the above expression, $k_{2}$ can be written as

$$
\begin{aligned}
k_{2}= & \frac{e^{2} \mu_{e g}^{2}}{48 \hbar \epsilon^{2} \pi^{2}} \int_{\frac{\hbar \Omega}{v_{f}}}^{\frac{2 E_{F}+\hbar \Omega}{v_{f}}} d q \frac{q^{3} e^{-2 q z}}{\sqrt{q^{2} v_{f}^{2}-(\hbar \Omega)^{2}}} \\
& \times \int_{1}^{\frac{2 E_{F}+\hbar \Omega}{q_{v}}} d \mu \sqrt{\mu^{2}-1} .
\end{aligned}
$$

We now make a change of the variable of integration from $q$ to $x$ defined by $q=\frac{\hbar \Omega}{v_{f}}+x$. Using this transformation, $k_{2}$ can be written as

$$
\begin{aligned}
k_{2}= & \frac{e^{2} \mu_{e g}^{2}}{48 \hbar \epsilon^{2} \pi^{2}} e^{\frac{-2 z \hbar \Omega}{v_{f}}} \int_{0}^{\frac{2 E_{F}}{v_{f}}} d x \frac{\left(\frac{\hbar \Omega}{v_{f}}+x\right)^{3} e^{-2 z x}}{\sqrt{x^{2} v_{f}^{2}+2 \hbar \Omega v_{f} x}} \\
& \times \int_{1}^{\frac{2 E_{F}+\hbar \Omega}{x v_{f}+\hbar \Omega}} d \mu \sqrt{\mu^{2}-1}
\end{aligned}
$$

For large values of $z$, in the above integral over $x$, because of the presence of $e^{-2 z x}$ term, only small values of $x$ are important. Therefore, for small values of $x$, the above expression can be simplified to get

$$
\begin{aligned}
k_{2}= & \sqrt{\frac{e^{4} \mu_{e g}^{4} \hbar^{3} \Omega^{5}}{\pi^{4} \epsilon^{4} v_{f}^{7}} \frac{e^{\frac{-2 z \hbar \Omega}{v_{f}}}}{48 \sqrt{2}} \int_{1}^{1+\frac{2 E_{F}}{\hbar \Omega}} d \mu \sqrt{\mu^{2}-1}} \\
& \times \int_{0}^{\frac{2 E_{F}}{v_{f}}} d x \frac{e^{-2 z x}}{\sqrt{x}} .
\end{aligned}
$$


The integral over $\mu$ can now be performed, and the upper limit in the integral over $x$ can be extended to $\infty$ to get the following expression for $k_{2}$ :

$$
\begin{aligned}
k_{2}= & \sqrt{\frac{e^{4} \mu_{e g}^{4} \hbar^{3} \Omega^{5}}{\pi^{3} \epsilon^{4} v_{f}^{7}}} \frac{e^{\frac{-2 z \hbar \Omega}{v_{f}}}}{192 \sqrt{z}} \\
& \times\left(r \sqrt{r^{2}-1}-\log \left[r+\sqrt{r^{2}-1}\right]\right),
\end{aligned}
$$

where $r=1+\frac{2 E_{F}}{\hbar \Omega}$.

2. Case II: We now consider the case $E_{F}>\frac{\hbar \Omega}{2}$. Therefore, $2 E_{F}-\hbar \Omega>0$ and hence $k_{2}$ is given by

$$
\begin{aligned}
k_{2}= & \frac{e^{2} \mu_{e g}^{2}}{48 \hbar \epsilon^{2} \pi^{2}} \int_{0}^{\infty} d q q^{3} e^{-2 q z} \frac{\Theta\left(q v_{f}-\hbar \Omega\right)}{\sqrt{q^{2} v_{f}^{2}-(\hbar \Omega)^{2}}} \\
& \times \int_{\operatorname{Max}\left[1, \frac{2 E_{F}-\hbar \Omega}{q v_{f}}\right]}^{\frac{2 E_{F} \hbar \Omega}{q v_{f}}} d \mu \sqrt{\mu^{2}-1} \Theta\left[\frac{2 E_{F}+\hbar \Omega}{q v_{f}}-1\right] .
\end{aligned}
$$

The above equation can be simplified to get

$$
\begin{aligned}
k_{2}= & \frac{e^{2} \mu_{e g}^{2}}{48 \hbar \epsilon^{2} \pi^{2}} \int_{\frac{\hbar \Omega}{v_{f}}}^{\frac{2 E_{F}+\hbar \Omega}{v_{f}}} d q \frac{q^{3} e^{-2 q z}}{\sqrt{q^{2} v_{f}^{2}-(\hbar \Omega)^{2}}} \\
& \times \int_{\operatorname{Max}\left[1, \frac{2 E_{F}-\hbar \Omega}{q v_{f}}\right]}^{\frac{2 E_{F}+\hbar \Omega}{q v_{f}}} d \mu \sqrt{\mu^{2}-1} .
\end{aligned}
$$

We now use the same procedure as was used for evaluating the integrals in Case I to get

$$
\begin{aligned}
& k_{2}= \sqrt{\frac{e^{4} \mu_{e g}^{4} \hbar^{3} \Omega^{5}}{\pi^{4} \epsilon^{4} v_{f}^{7}}} \frac{e^{\frac{-2 z_{f}}{v_{f}}}}{48 \sqrt{2}} \\
& \quad \times \int_{\operatorname{Max}\left[1, \frac{2 E_{F}}{\hbar \Omega}-1\right]}^{1+\frac{2 E_{F}}{\hbar \Omega}} d \mu \sqrt{\mu^{2}-1} \int_{0}^{\frac{2 E_{F}}{v_{f}}} d x \frac{e^{-2 z x}}{\sqrt{x}} .
\end{aligned}
$$

On evaluating the integral over $\mu$, we get

$$
\begin{aligned}
k_{2}= & \sqrt{\frac{e^{4} \mu_{e g}^{4} \hbar^{3} \Omega^{5}}{\pi^{4} \epsilon^{4} v_{f}^{7}}} \frac{e^{\frac{-2 \hbar \hbar \Omega}{v_{f}}}}{96 \sqrt{2}}\left(-s \sqrt{s^{2}-1}+r \sqrt{r^{2}-1}\right. \\
& \left.+\log \left[s+\sqrt{s^{2}-1}\right]-\log \left[r+\sqrt{r^{2}-1}\right]\right) \\
& \times \int_{0}^{\frac{2 E_{F}}{v_{f}}} d x \frac{e^{-2 z x}}{\sqrt{x}}
\end{aligned}
$$

where $s=\operatorname{Max}\left[1, \frac{2 E_{F}}{\hbar \Omega}-1\right]$ and $r=1+\frac{2 E_{F}}{\hbar \Omega}$. For $E_{F}$ close to $\frac{\hbar \Omega}{2}, s=1$. Using this and extending the upper limit of the integral over $x$ to $\infty$ and then evaluating the integral, we get

$$
k_{2}=\sqrt{\frac{e^{4} \mu_{e g}^{4} \hbar^{3} \Omega^{5}}{\pi^{3} \epsilon^{4} v_{f}^{7}}} \frac{e^{\frac{-22 \hbar \Omega}{v_{f}}}}{192 \sqrt{z}}
$$

$$
\times\left(r \sqrt{r^{2}-1}-\log \left[r+\sqrt{r^{2}-1}\right]\right) .
$$

\section{References}

1. Swathi R S and Sebastian K L 2008 J. Chem. Phys. 129, 054703

2. Swathi R S and Sebastian K L 2009a J. Chem. Phys. 130, 086101

3. Swathi R S and Sebastian K L 2009b J. Chem. Sci. 121, 777

4. Kim J, Cote L J, Kim F and Huang J 2010 J. Am. Chem. Soc. 132, 260

5. Xie L, Ling X, Fang Y, Zhang J and Liu Z 2009 J. Am. Chem. Soc. 131, 9890

6. Sagar A, Kern K and Balasubramanian K 2010 Nanotechnology 21, 015303

7. He S, Song B, Li D, Zhu C, Qi W, Wen Y, Wong L, Song S, Fang H and Fan C 2010 Adv. Funct. Mater. 20, 453

8. Chang H, Tang L, Wang Y, Jiang J and Li $2010 \mathrm{~J}$. Anal. Chem. 82, 2341

9. Swathi R S and Sebastian K L 2010 J. Chem. Phys. 132, 104502

10. Wong C Y, Curutchet C, Tretiak S and Scholes G D 2009 J. Chem. Phys. 130, 081104

11. Wong K F, Bagchi B and Rossky P J 2004 J. Phys. Chem. A 108, 5752

12. Achermann M, Petruska M A, Kos S, Smith D L, Koleske D D and Klimov V I 2004 Nature 429, 642

13. Schedin F, Geim A K, Morozov S V, Hill E W, Blake P, Katsnelson M I and Novoselov K S 2007 Nat. Mater. 6, 652

14. Bostwick A, Ohta T, Seyller T, Horn K and Rotenberg E 2007 Nat. Phys. 3, 36

15. Swathi R S 2010 PhD Thesis, Department of Inorganic and Physical Chemistry, Indian Institute of Science, Bangalore

16. Swathi R S and Sebastian K L 2011 arXiv:1104.2708v1

17. Yanagi K, Lakoubovskii K, Matsui H, Matsuzaki H, Okamoto H, Miyata Y, Maniwa Y, Kazaoui S, Minami N and Kataura H 2007 J. Am. Chem. Soc. 129, 4992

18. Velizhanin K A and Efimov A 2011 arXiv: $1104.0233 \mathrm{v} 1$ 